\title{
Cholesterol binding, efflux, and a PDZ-interacting domain of scavenger receptor-BI mediate HDL-initiated signaling
}

Chatchawin Assanasen, ${ }^{1}$ Chieko Mineo,, Divya Seetharam,, Ivan S. Yuhanna, ${ }^{1}$ Yves L. Marcel, ${ }^{2}$ Margery A. Connelly, ${ }^{3}$ David L. Williams, ${ }^{3}$ Margarita de la Llera-Moya, ${ }^{4}$ Philip W. Shaul, ${ }^{1}$ and David L. Silver ${ }^{5}$

\author{
1Department of Pediatrics, University of Texas Southwestern Medical Center, Dallas, Texas, USA. ${ }^{2}$ Faculty of Medicine \\ University of Ottawa Heart Institute, Ottawa, Ontario, Canada. ${ }^{3}$ Department of Pharmacological Sciences, University Medical Center, \\ State University of New York at Stony Brook, Stony Brook, New York, USA. ${ }^{4}$ Division of GI/Nutrition, Lipid Research Group, \\ The Children's Hospital of Philadelphia, University of Pennsylvania School of Medicine, Philadelphia, Pennsylvania, USA. \\ ${ }^{5}$ Department of Medicine, Division of Molecular Medicine, Columbia University College of Physicians and Surgeons, New York, New York, USA.
}

\begin{abstract}
The binding of HDL to scavenger receptor-BI (SR-BI) mediates cholesterol movement. HDL also induces multiple cellular signals, which in endothelium occur through SR-BI and converge to activate eNOS. To determine the molecular basis of a signaling event induced by HDL, we examined the proximal mechanisms in HDL activation of eNOS. In endothelial cells, HDL and methyl- $\beta$-cyclodextrin caused comparable eNOS activation, whereas cholesterol-loaded methyl- $\beta$-cyclodextrin had no effect. Phosphatidylcholine-loaded HDL caused greater stimulation than native HDL, and blocking antibody against SR-BI, which prevents cholesterol efflux, prevented eNOS activation. In a reconstitution model in COS-M6 cells, wild-type SR-BI mediated eNOS activation by both HDL and small unilamellar vesicles (SUVs), whereas the SR-BI mutant AVI, which is incapable of efflux to SUV, transmitted signal by only HDL. In addition, eNOS activation by methyl- $\beta$ cyclodextrin was SR-BI dependent. Studies of mutant and chimeric class B scavenger receptors revealed that the C-terminal cytoplasmic PDZ-interacting domain and the C-terminal transmembrane domains of SR-BI are both necessary for HDL signaling. Furthermore, we demonstrated direct binding of cholesterol to the C-terminal transmembrane domain using a photoactivated derivative of cholesterol. Thus, HDL signaling requires cholesterol binding and efflux and C-terminal domains of SR-BI, and SR-BI serves as a cholesterol sensor on the plasma membrane.
\end{abstract}

\section{Introduction}

Circulating levels of HDL are inversely related to the incidence of atherosclerosis, and clinical trials with agents that increase HDL show that elevations in the lipoprotein level decrease cardiovascular disease risk $(1,2)$. Thus, HDL is not merely a marker but a potent mediator of cardiovascular health. The HDL particle consists of a shell of apolipoproteins (mainly apolipoproteins apoA-I and apoA-II), phospholipids, and cholesterol surrounding a lipid core of triglycerides and cholesteryl esters. Atheroprotection by HDL is in part due to the action of HDL in delivering extrahepatic cholesterol to the liver for excretion into bile in a process known as reverse cholesterol transport. Reverse cholesterol transport involves the binding of HDL to hepatic scavenger receptor-BI (SR-BI), an 82-kDa cell surface glycoprotein. SR-BI is also expressed at high levels in the adrenal glands and gonads, where it participates in regulating steroid hormone synthesis $(3,4)$.

Nonstandard abbreviations used: BAEC, bovine aortic endothelial cell; CD, methyl$\beta$-cyclodextrin; LP2A-I, lipoprotein containing 2 molecules of apolipoprotein A-I; NPC1, Niemann-Pick C1; PC, phosphatidylcholine; PC-HDL, PC-loaded HDL; POPC, palmitoyloleoylphosphatidylcholine; SCAP, SREBP cleavage-activating protein; S1P, sphingosine-1-phosphate; SR-BI, scavenger receptor-BI; StAR, steroidogenic acute regulatory protein; START, StAR-related lipid transfer; SUV, small unilamellar vesicle. Conflict of interest: The authors have declared that no conflict of interest exists.

Citation for this article: J. Clin. Invest. 115:969-977 (2005)

doi:10.1172/JCI200523858
SR-BI was first identified by its sequence homology to the class B scavenger receptor CD36 $(5,6)$. CD36 and SR-BI share approximately $30 \%$ amino acid sequence homology, and the predicted secondary structures are nearly identical, with each protein comprising 2 transmembrane and 2 cytoplasmic domains and a large extracellular domain. SR-BI plays a key role in HDL metabolism by facilitating cholesteryl ester transport from HDL to cells in a process termed selective uptake. SR-BI also stimulates the bidirectional flux of free cholesterol between cells and lipoproteins. In addition, SR-BI mediates the increase in cellular cholesterol mass and alters cholesterol distribution in plasma membrane domains, as judged by the enhanced sensitivity of membrane cholesterol to extracellular cholesterol oxidase $(7,8)$. Previous studies have shown that these key lipid transport functions of SR-BI are dependent on the extracellular domain of the receptor (9).

Although the atheroprotection afforded by HDL and SR-BI have been previously attributed to reverse cholesterol transport, the mechanisms underlying the antiatherogenic properties of the lipoprotein are not well understood (10). Recent studies indicate that in addition to its capacity to mediate cellular and global cholesterol metabolism, HDL initiates signaling in a variety of cell types relevant to atherogenesis, including endothelium, vascular smooth muscle, monocytes, and platelets $(11,12)$. These signaling events include the activation of PKC, phosphatidylinositol- and phosphatidylcholine-specific (PC-specific) phospholipases C and D, MAPK, tyrosine kinase, heterotrimeric G proteins, and $\mathrm{p} 21^{\text {ras; }}$ 

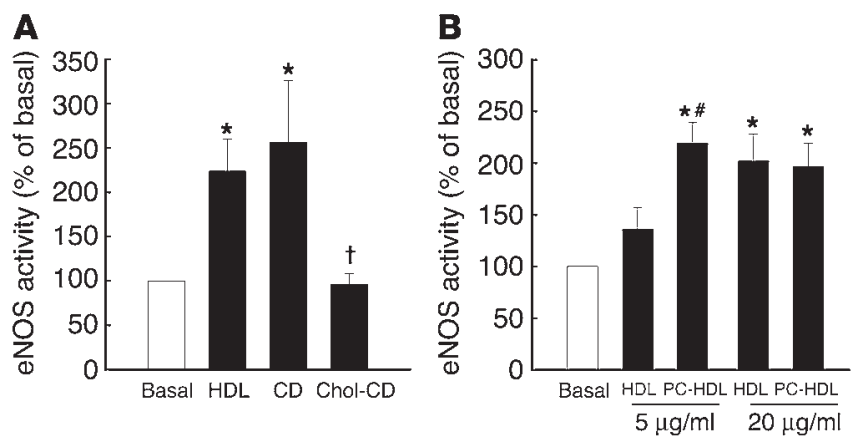

Figure 1

Cholesterol efflux is required for HDL stimulation of eNOS. (A) eNOS activation was assessed in BAECs by measuring $\left[{ }^{3} \mathrm{H}\right] \mathrm{L}$-arginine to $\left[{ }^{3} \mathrm{H}\right] \mathrm{L}$-citrulline conversion during 15 -minute incubations with control buffer (Basal), HDL (50 $\mu \mathrm{g} / \mathrm{ml}), \mathrm{CD}(2 \%)$, or cholesterol-loaded CD (Chol-CD, $2 \%)$. (B) eNOS activation in BAECs was assessed during 15-minute incubations with control buffer, HDL (5 and $20 \mu \mathrm{g} / \mathrm{ml}$ ), or PC-HDL (5 and $20 \mu \mathrm{g} / \mathrm{ml}$ ). Values (mean \pm SEM) are expressed relative to basal activity, designated as $100 \% ; n=4 .{ }^{*} P<0.05$ versus basal; ${ }^{\dagger} P<0.05$ versus no cholesterol. ${ }^{\#} P<0.05$ versus HDL.

the production of cAMP and ceramide; and intracellular calcium release (11). We have previously demonstrated that HDL causes potent activation of eNOS in vascular endothelium through the binding of apoA-I to SR-BI (13), thereby generating the antiatherogenic signaling molecule NO. In endothelium, both eNOS and SR-BI are localized to cholesterol-enriched plasma membrane domains known as caveolae, which are a subset of lipid rafts. Studies with isolated caveolae membranes indicate that all of the molecular machinery coupling SR-BI to eNOS resides within the domain (13). In recent work, we have further demonstrated that HDL stimulation of eNOS is mediated by the activation of Src family kinase(s), PI3K, Akt kinase, and MAPK and the phosphorylation of eNOS at Ser1179 (14). Thus, HDL induces multiple signaling processes that are likely to be critical to the atheroprotective features of the lipoprotein. However, the most proximal events underlying HDL-initiated signaling are yet to be elucidated.
The purpose of the present study was to determine the molecular basis of a signaling event induced by HDL via SR-BI by investigating the proximal mechanisms in HDL activation of eNOS. Experiments were designed to delineate whether signaling requires the recognized function of HDL to cause cholesterol flux to or from cells and to delimit the structural features of SR-BI necessary for signal transduction.

\section{Results}

Cholesterol flux and HDL signaling. To examine the role of cholesterol flux in HDL-mediated eNOS stimulation, we compared the effects of methyl- $\beta$-cyclodextrin (CD) and cholesterol-loaded CD with those of native HDL in bovine aortic endothelial cells (BAECs). As shown in Figure 1A, 15-minute incubation with cholesterol-free CD induced eNOS activation to the same extent as incubation with HDL. Incubation with CD for 15 minutes did not displace eNOS from the plasma membrane (data not shown), which is an event that occurs if endothelial cells are treated with CD for a longer duration (15). In contrast to cholesterol-free CD, cholesterol-loaded CD did not stimulate eNOS activation, which indicates that cholesterol efflux is important for eNOS activation. We further tested the role of cholesterol efflux to HDL in this process using PC-loaded HDL (PC-HDL) (Figure 1B); the addition of PC to HDL increases efflux to the lipoprotein (16). PC-HDL at $5 \mu \mathrm{g} / \mathrm{ml}$ was capable of stimulating eNOS, whereas native HDL was not. At a higher concentration $(20 \mu \mathrm{g} / \mathrm{ml})$, native HDL and PC-HDL yielded similar stimulation. In additional experiments, cholesterol-free Lp2A-I (lipoprotein containing 2 molecules of apolipoprotein A-I) particles consisting of apoA-I and palmitoyloleoylphosphatidylcholine (POPC) caused eNOS activation in BAECs comparable to that induced by native HDL (Figure 2A); variations in POPC content yielded changes in the degree of signaling that paralleled previously demonstrated alterations in efflux capacity (Figure $2 \mathrm{~B})(17,18)$; and the addition of cholesterol to Lp2A-I prevented signaling (Figure $2 \mathrm{C}$ ). Taken together, the data indicate that particles that readily accept cellular cholesterol activate eNOS, whereas those that serve as cholesterol donors do not.

We also manipulated SR-BI to assess the role of efflux in HDL signaling. The effect of SR-BI-neutralizing antibody, which binds to the extracellular domain of SR-BI and prevents SR-BI-mediated
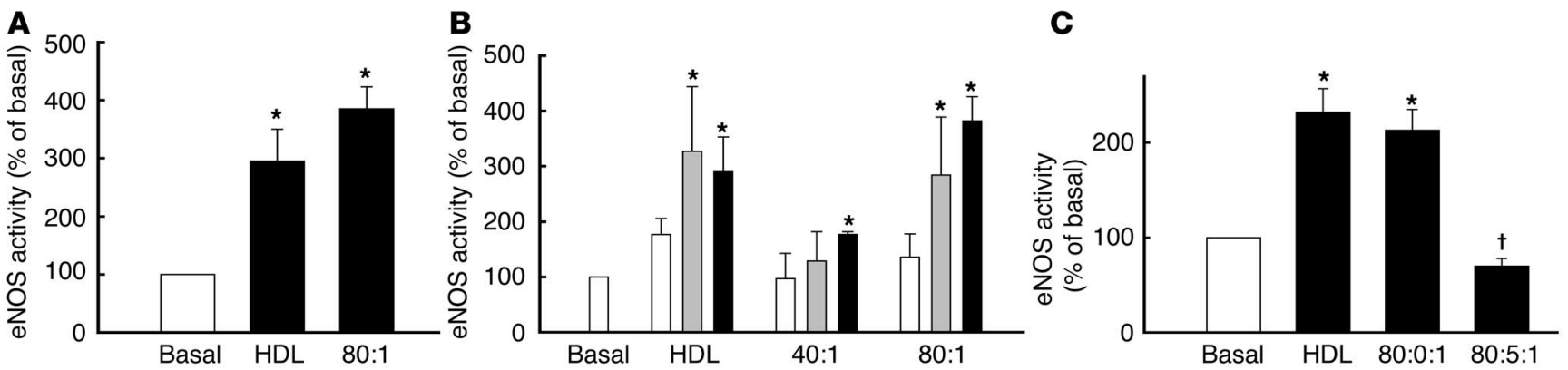

Figure 2

Cholesterol-free Lp2A-I particles activate eNOS. (A) eNOS activation in BAECs was assessed by measuring $\left[{ }^{3} \mathrm{H}\right] \mathrm{L}-\mathrm{arginine}$ to $\left[{ }^{3} \mathrm{H}\right] \mathrm{L}-\mathrm{citrul}$ line conversion during 15-minute incubations with control buffer, HDL $(20 \mu \mathrm{g} / \mathrm{ml})$, or Lp2A-I particles with a molar POPC/apoA-I ratio of 80:1 $(20 \mu \mathrm{g} / \mathrm{ml})$. (B) eNOS activation in BAECs was assessed during 15-minute incubations with control buffer, HDL (1, 5, and 20 $\mu \mathrm{g} / \mathrm{ml}), \mathrm{Lp} 2 \mathrm{~A}-\mathrm{I}$ particles with a molar POPC/apoA-I ratio of $40: 1(1,5$, and $20 \mu \mathrm{g} / \mathrm{ml})$, or Lp2A-I particles with a molar POPC/apoA-I ratio of 80:1 (1, 5, and $20 \mu \mathrm{g} / \mathrm{ml}$ ). White bars, $1 \mu \mathrm{g} / \mathrm{ml}$; gray bars, $5 \mu \mathrm{g} / \mathrm{ml}$; black bars, $20 \mu \mathrm{g} / \mathrm{ml}$. (C) eNOS activation in BAECs was assessed during 15-minute incubations with control buffer, HDL $(20 \mu \mathrm{g} / \mathrm{ml})$, Lp2A-I particles with a molar POPC/cholesterol/apoA-I ratio of 80:0:1 (20 $\mu \mathrm{g} / \mathrm{ml})$, or Lp2A-I particles with a molar POPC/cholesterol/apoA-I ratio of $80: 5: 1(20 \mu \mathrm{g} / \mathrm{ml})$. Values (mean $\pm \mathrm{SEM}$ ) are expressed relative to basal activity designated as $100 \%$; $n=4$. ${ }^{*} P<0.05$ versus basal; $\uparrow P<0.05$ versus no cholesterol. 
A
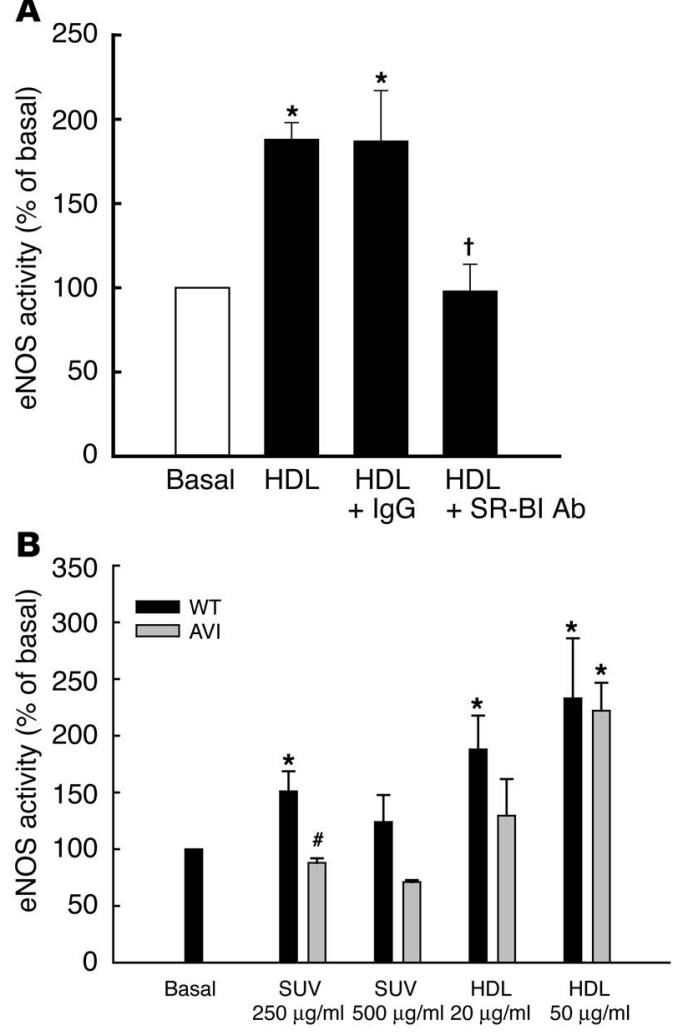

cholesterol efflux (19), was tested in BAECs (Figure 3A). In cells preincubated with neutralizing antibody, HDL failed to stimulate eNOS, whereas preincubation with control IgG had no effect. In contrast, $\mathrm{CD}$ activation of eNOS was similar in the absence or presence of neutralizing antibody (stimulation to $204 \% \pm 34 \%$ and $188 \% \pm 20 \%$ of basal activity, respectively), which is consistent with CD function being independent of binding to SR-BI $(20,21)$. To further establish a role for cholesterol efflux, experiments were performed with native HDL and small unilamellar vesicles (SUVs) in COS-M6 cells expressing eNOS and either wild-type SR-BI or the AVI mutant of SR-BI (Figure 3B). Whereas the wild-type receptor mediates efflux to either HDL or SUV, the AVI mutant is capable of efflux to HDL but not to SUV (22). The activation of eNOS by HDL and SUVs paralleled their induction of cholesterol efflux: HDL activated eNOS via either wild-type SR-BI or the AVI mutant, and SUVs caused eNOS stimulation exclusively in cells expressing wild-type receptor.

$S R-B I$ and HDL signaling. To further determine the specific role of SR-BI, we compared the ability of CD36 and SR-BI to transmit HDL signaling in COS-M6 cells expressing eNOS. HDL binds with the

\section{Figure 4}

$\mathrm{SR}-\mathrm{BI}$ is required for HDL-mediated eNOS activation. (A) COS-M6 cells were transfected with eNOS cDNA and either SR-BI or CD36 cDNA. Forty-eight hours after transfection, eNOS activation was assessed by measuring $\left[{ }^{3} \mathrm{H}\right] \mathrm{L}$-arginine to $\left[{ }^{3} \mathrm{H}\right] \mathrm{L}$-citrulline conversion during 15 -minute incubations with control buffer or HDL $(50 \mu \mathrm{g} / \mathrm{ml})$. ${ }^{*} P<0.05$ versus basal; ${ }^{\dagger} P<0.05$ versus SR-BI. (B) COS-M6 cells were transfected with eNOS cDNA and either sham plasmid or SR-BI cDNA. Forty-eight hours after transfection, eNOS activation was assessed during 15-minute incubations with control buffer, $\mathrm{HDL}(50 \mu \mathrm{g} / \mathrm{ml})$, or CD $(2 \%) .{ }^{*} P<0.05$ versus basal; $\#<0.05$ versus sham. Values (mean \pm SEM) are expressed relative to basal activity designated as $100 \% ; n=4$.

\section{Figure 3}

Interventions that decrease SR-BI efflux capacity attenuate eNOS stimulation. (A) BAECs were preincubated with control IgG or SR-BI blocking antibody for 30 minutes, and eNOS activation was assessed during 15-minute incubations with control buffer or HDL $(50 \mu \mathrm{g} / \mathrm{ml})$. (B) COS-M6 cells were transfected with eNOS cDNA and either wildtype SR-BI or AVI mutant SR-BI cDNA. Forty-eight hours following transfection, eNOS activation was assessed during 15-minute incubations with control buffer, HDL (20 and $50 \mu \mathrm{g} / \mathrm{ml}$ ), or SUVs (250 or $500 \mu \mathrm{g} / \mathrm{ml}$ ). Values (mean \pm SEM) are expressed relative to basal activity designated as $100 \% ; n=4 .{ }^{*} P<0.05$ vs. basal; ${ }^{\dagger} P<0.05$ versus no SR-BI Ab; ${ }^{P} P<0.05$ versus wild-type SR-BI.

same affinity to both SR-BI and CD36, but CD36 induces approximately $75 \%$ less cholesterol efflux compared with SR-BI $(7,9)$. Whereas SR-BI yielded eNOS activation upon HDL binding, CD36 did not (Figure 4A).

To test whether SR-BI is required for eNOS activation in response to cholesterol efflux independent of its capacity to bind HDL, we assessed eNOS activation by CD in COS-M6 cells devoid of or expressing SR-BI. As shown in Figure 4B, both HDL-related and CD-related activation of eNOS were SR-BI dependent.

$S R-B I C$-terminal cytoplasmic domain and HDL signaling. In previous work delineating how SR-BI transduces signaling by HDL to activate eNOS, the addition of an antibody against the C-terminal cytoplasmic domain of the receptor inhibited eNOS activation by HDL in isolated plasma membranes (13). To further study the role of the C-terminal cytoplasmic domain, we tested the ability of SR-BII to mediate eNOS phosphorylation and enzyme activation in COS-M6 cells expressing eNOS (Figure 5). SR-BII is a splice variant of SR-BI that is identical in structure except for the C-terminal cytoplasmic domain, and SR-BII displays HDL binding and cholesterol flux similar to those of SR-BI (23). In contrast to SR-BI, SR-BII did not stimulate eNOS phosphorylation (Figure 5, top) or eNOS enzymatic activity (Figure 5, bottom). The extreme C-terminus of
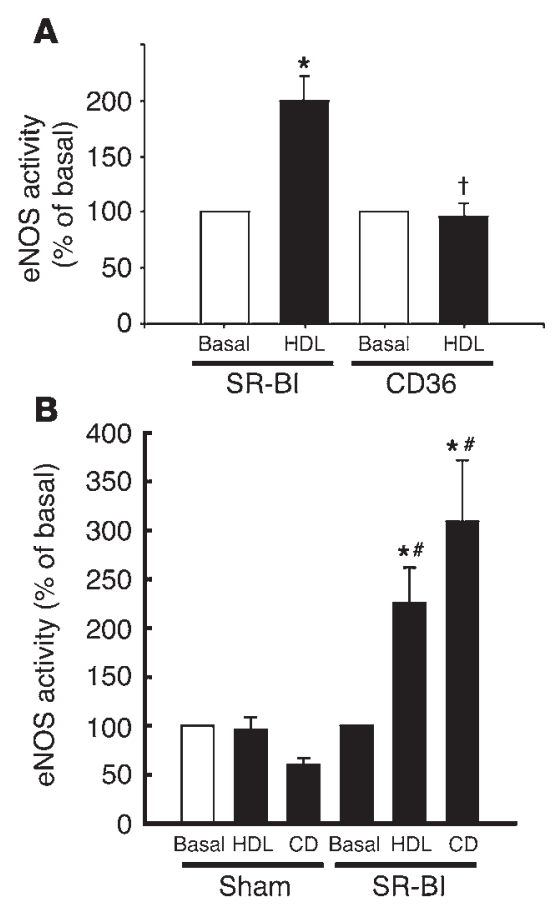


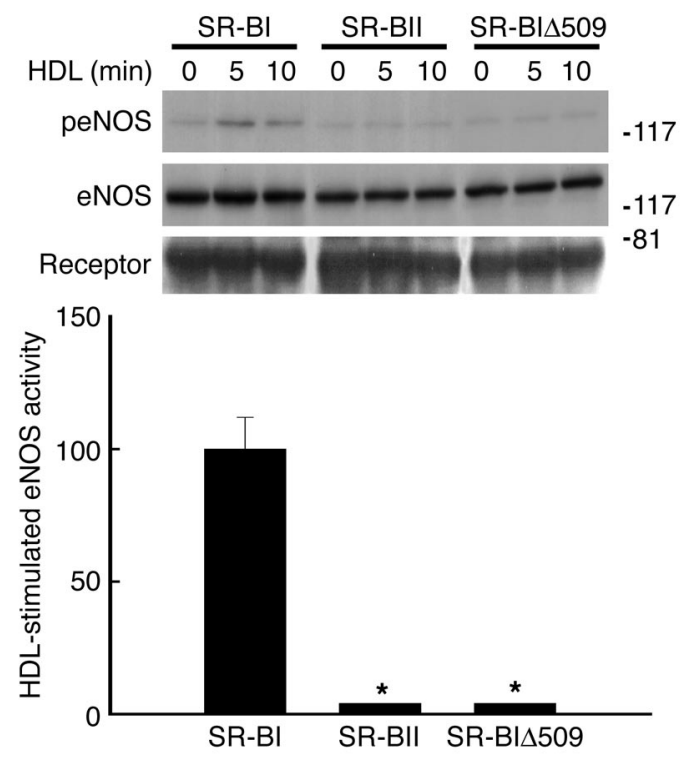

SR-BI was recently shown to contain a PDZ-interacting domain that is essential for binding to the PDZ domain-containing protein PDZK1 (24). A mutant of the receptor lacking the last amino acid (SR-BI $\triangle 509$ ) was used to test the role of the PDZK1-binding domain (24). In contrast to wild-type receptor, SR-BI $\Delta 509$ did not cause eNOS phosphorylation or activation. Additional experiments indicated that eNOS localization in caveolae was not altered by SR-BI, SR-BII, or SR-BIA509 (data not shown).

SR-BI C-terminal transmembrane domain and HDL signaling. To determine whether other domains of SR-BI in addition to the C-terminal cytoplasmic domain are required for HDL signaling, we studied SR-BI and CD36 chimeras in COS-M6 cells expressing eNOS. In one chimera consisting primarily of CD36 sequence, the SR-BI C-terminal cytoplasmic domain added to the C-terminus of CD36 (CD/SRCT; Figure 6). A second chimera consisting primarily of CD36 contained both the C-terminal transmembrane and cytoplasmic domains of SR-BI (CD/SRCTMT; Figure 6). As expected based on the findings reported in Figure 4A, wild-type SR-BI caused eNOS phosphorylation and activation in response to HDL, and wild-type CD36 did not (Figure 7A). While containing the SR-BI PDZ-interacting domain essential for eNOS activation (Figure 5), CD/SRCT failed to cause eNOS phosphorylation or activation, which indicates a requirement for other domains of SR-BI. Surprisingly, the addition of the C-terminal transmembrane domain to CD/SRCT in the chimera designated CD/SRCTMT yielded eNOS phosphorylation and activation comparable to those of wild-type SR-BI. The cell surface expression of both CD/SRCT and CD/SRCTMT was comparable to that of SR-BI and CD36, and their capacity to cause cholesterol efflux mimicked that of CD36 (data not shown).

Our data indicate that the C-terminal PDZ-interacting domain of SR-BI is essential for transmitting signals to activate eNOS in response to cholesterol efflux from the plasma membrane (Figure 5). Furthermore, the C-terminal transmembrane domain of SR-BI is also required for this function (Figure 7A). Based on prior work

\section{Figure 6}

Schematic diagram of SR-BI, CD36, and chimeric receptors. The $\mathrm{SR}-\mathrm{BI}-$ derived sequence is shown in gray, and the CD36-derived sequence is shown in black.
Figure 5

SR-BI C-terminal cytoplasmic domain is required for eNOS phosphorylation and activation. COS-M6 cells were transfected with eNOS cDNA and either wild-type SR-BI, SR-BII, or SR-BI 5509 cDNA. For detection of eNOS phosphorylation (top), 24 hours after transfection the cells were starved in serum-free DMEM for another 24 hours, the cells were incubated with HDL $(50 \mu \mathrm{g} / \mathrm{ml})$ for $0-10$ minutes, and cell lysates were analyzed by Western blotting using anti-phospho-eNOSspecific (Ser1179-specific) antibody (peNOS), anti-eNOS monoclonal antibody (eNOS), and anti-SR-BI (extracellular domain) polyclonal antibody (Receptor). For detection of eNOS activation (bottom), parallel sets of transfected cells were used, and $\left[{ }^{3} \mathrm{H}\right] \mathrm{L}$-arginine conversion to $\left[{ }^{3} \mathrm{H}\right] \mathrm{L}$-citrulline was measured during 15-minute incubations with control buffer or HDL $(50 \mu \mathrm{g} / \mathrm{ml})$. Values (mean \pm SEM) are expressed as percent of HDL-stimulated activity above basal activity; $n=4$. ${ }^{*} P<0.05$ versus wild-type SR-BI.

indicating that sterol sensing by HMG-CoA reductase, SREBP cleavage-activating protein (SCAP), and Niemann-Pick C1 (NPC1) entails cholesterol binding to transmembrane domains within these proteins (25-29), the hypothesis was raised that the C-terminal transmembrane domain of SR-BI binds cholesterol. The ability of full-length, wild-type SR-BI versus CD/SRCT to bind cholesterol was tested in HEK 293 cells expressing the receptors, which were labeled with the photoactivatable, crosslinkable cholesterol analog $\left[{ }^{3} \mathrm{H}\right] 6$-photocholesterol. Similar strategies were recently employed to demonstrate direct cholesterol binding to SCAP (30). Cells were UV irradiated, SR-BI or CD/SRCT was immunoprecipitated, associated photocholesterol was assessed by autoradiography (Figure 7B, top), and the abundance of immunoprecipitated receptor was deter-
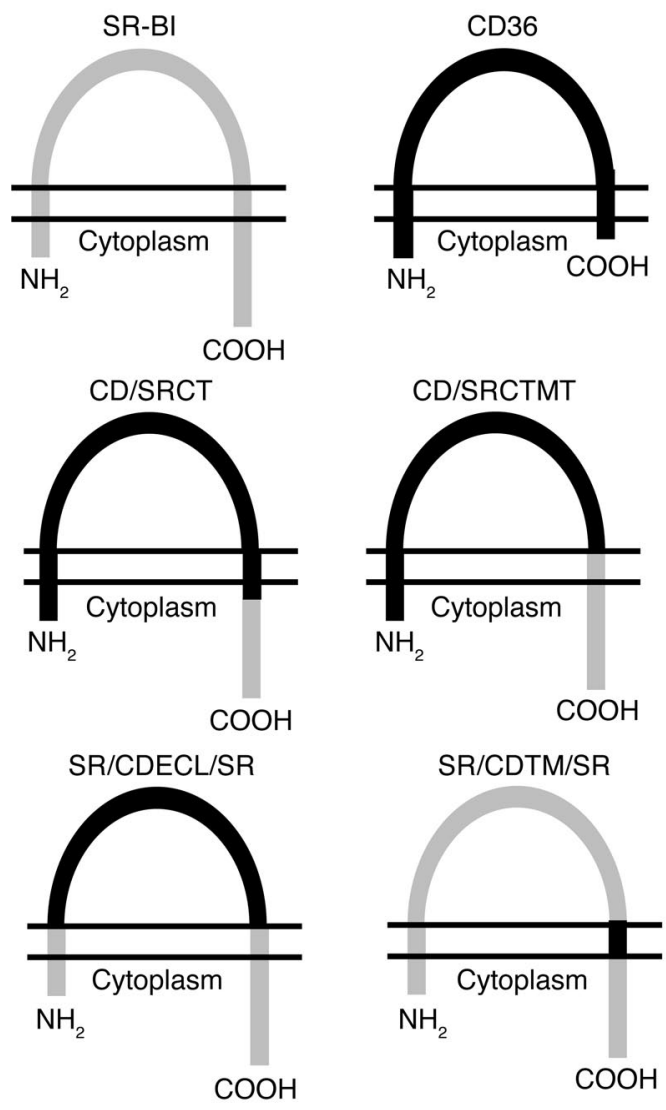
A

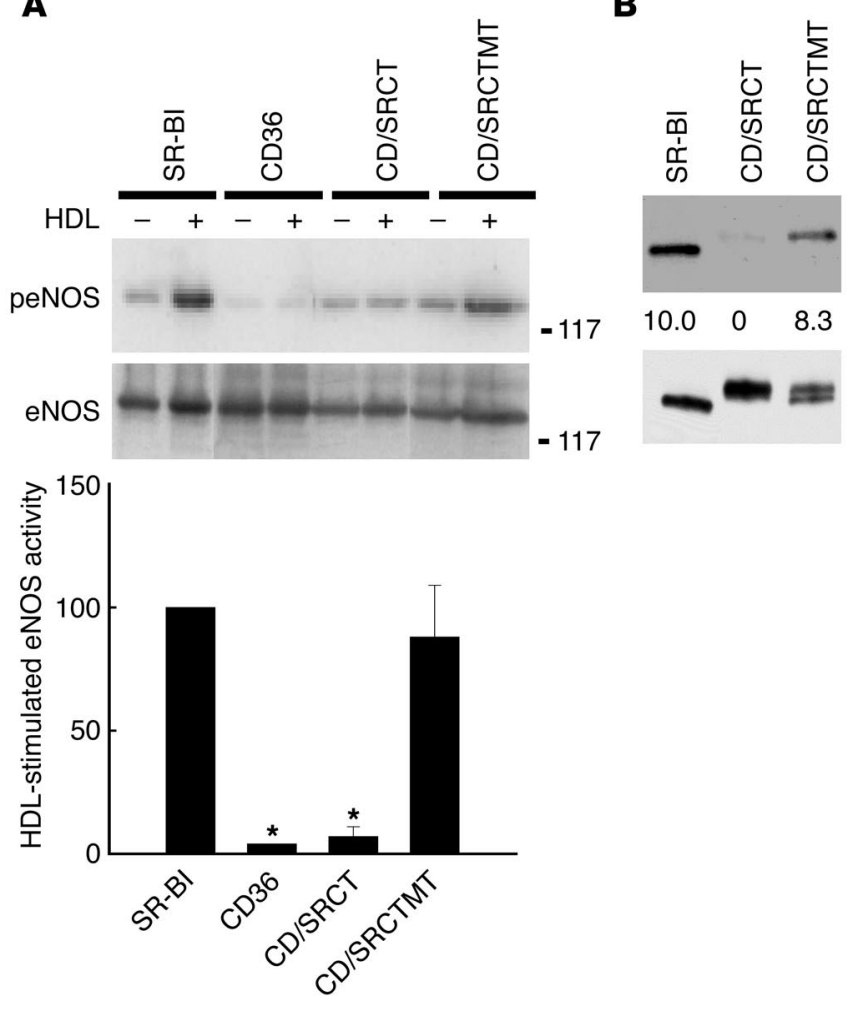

\section{Figure 7}

SR-BI C-terminal cytoplasmic domain is not sufficient for eNOS activation, and the $\mathrm{C}$-terminal transmembrane domain, which binds cholesterol, is also required. (A) COS-M6 cells were transfected with eNOS cDNA and either wild-type SR-BI, CD36, CD/SRCT, or CD/SRCTMT cDNA. For detection of eNOS phosphorylation (top), 24 hours after transfection, the cells were starved in serum-free DMEM for another 24 hours and incubated with HDL $(50 \mu \mathrm{g} / \mathrm{ml})$ for 0 or 10 minutes, and cell lysates were analyzed by Western blotting using anti-phosphoeNOS-specific (Ser1179-specific) antibody and anti-eNOS monoclonal antibody. For detection of eNOS activation (bottom), parallel sets of transfected cells were used, and $\left[{ }^{3} \mathrm{H}\right] \mathrm{L}$-arginine conversion to $\left[{ }^{3} \mathrm{H}\right] \mathrm{L}$-citrulline was measured during 15 -minute incubations with control buffer or HDL $(50 \mu \mathrm{g} / \mathrm{ml})$. Values (mean \pm SEM) are expressed as percent of HDL-stimulated activity above basal activity; $n=4$. ${ }^{*} P<0.05$ versus wild-type SR-BI. (B) HEK 293 cells were transfected and labeled with $\left[{ }^{3} \mathrm{H}\right]$ photocholesterol; receptors were immunoprecipitated with antibody to the SR-BI C-terminal tail; and the $\left[{ }^{3} \mathrm{H}\right]$ photocholesterol associated with the receptor was assessed by autoradiography (top). The abundance of immunoprecipitated receptor was assessed by Western blotting (bottom), and the relative amount of $\left[{ }^{3} \mathrm{H}\right]$ photocholesterolbound receptor versus total receptor was calculated.

with CD36 extracellular domain (SR/CDECL/SR) and SR-BI with CD36 C-terminal transmembrane domain (SR/CDTM/SR) were tested for eNOS phosphorylation and enzyme activation (Figure 9A). SR/CDECL/SR binds HDL with affinity similar to that of wild-type SR-BI, but it induces cholesterol efflux at $25 \%$ the level of wild-type SR-BI and similarly to wild-type CD36 (32). Wild-type SR-BI caused eNOS phosphorylation and activation in response to HDL, C-terminally tagged CD36 did not, and $\mathrm{SR} / \mathrm{CDECL} / \mathrm{SR}$ caused both signaling events. In contrast, SR/CDTM/SR, which mediates cholesterol efflux comparably to SR-BI (data not shown), did not phosphorylate or activate eNOS.

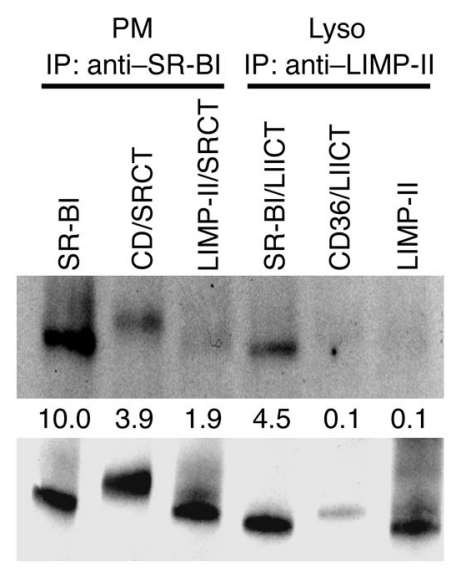

\section{Figure 8}

SR-BI binds either plasma membrane or lysosomal membrane cholesterol. HEK 293 cells were transfected with SR-BI, CD/SRCT, LIMP-II/SRCT, SR-BI/LIICT, CD36/LIICT, or LIMP-II and labeled with $\left[{ }^{3} \mathrm{H}\right]$ photocholesterol; plasma membranes or lysosomal membranes were isolated; receptors were immunoprecipitated with antibody to the SR-BI C-terminal tail or to LIMP-II; and the [ $\left.{ }^{3} \mathrm{H}\right]$ photocholesterol associated with the receptor was assessed by autoradiography (top). The abundance of immunoprecipitated receptor was assessed by Western blotting (bottom), and the relative amount of $\left[{ }^{3} \mathrm{H}\right]$ photocholesterolbound receptor versus total receptor was calculated. 
A

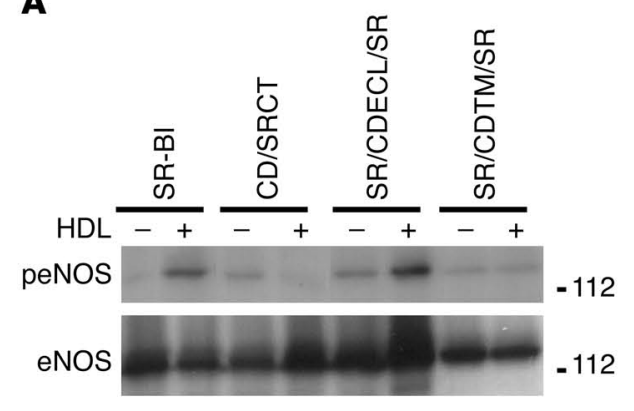

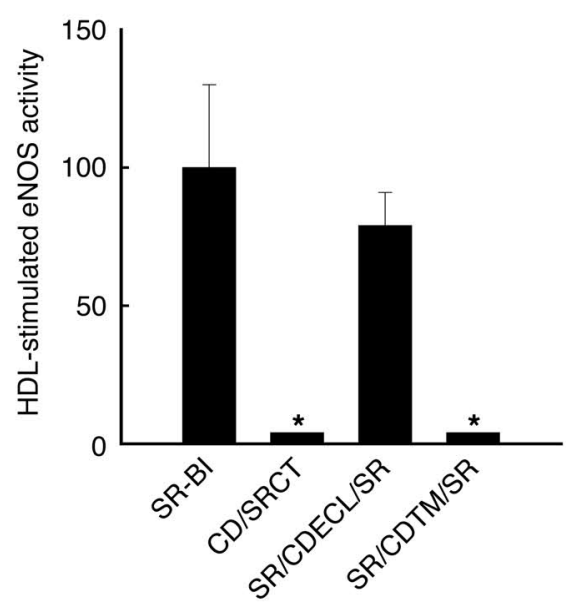

B

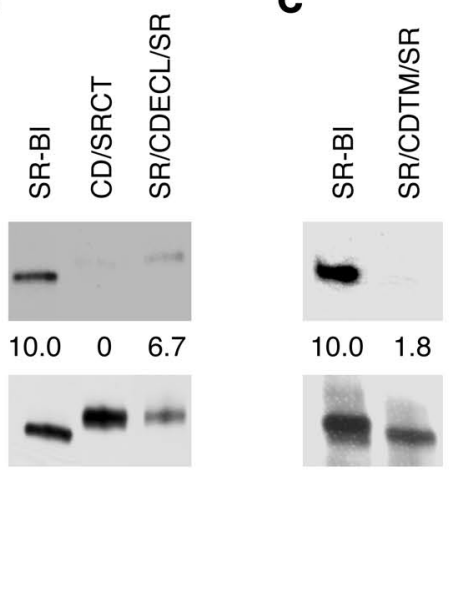

Figure 9

CD36 extracellular domain is capable of transducing signal if SR-BI transmembrane and cytoplasmic domains are present. (A) COS-M6 cells were transfected with eNOS cDNA and either wild-type SR-BI, CD/SRCT, SR/CDECL/SR, or SR/CDTM/SR cDNA. For detection of eNOS phosphorylation (left), 24 hours after transfection the cells were starved in serum-free DMEM for another 24 hours, the cells were incubated with HDL $(50 \mu \mathrm{g} / \mathrm{ml})$ for 0 or 10 minutes, and cell lysates were analyzed by Western blotting using anti-phospho-eNOS-specific (Ser1179specific) antibody and anti-eNOS monoclonal antibody. For detection of eNOS activation (right), parallel sets of transfected cells were used, and $\left[{ }^{3} \mathrm{H}\right] \mathrm{L}$-arginine conversion to $\left[{ }^{3} \mathrm{H}\right] \mathrm{L}$-citrulline was measured during 15 -minute incubations with control buffer or $\mathrm{HDL}(50 \mu \mathrm{g} / \mathrm{ml})$. Values (mean \pm SEM) are expressed as percent of HDL-stimulated activity above basal activity; $n=4$. ${ }^{*} P<0.05$ versus wild-type SR-BI. (B and C) HEK 293 cells were transfected with the constructs shown in A and labeled with [ $\left.{ }^{3} \mathrm{H}\right]$ photocholesterol; receptors were immunoprecipitated with antibody to the SR-BI C-terminal tail; and the $\left[{ }^{3} \mathrm{H}\right]$ photocholesterol associated with the receptor was assessed by autoradiography (top). The abundance of immunoprecipitated receptor was assessed by Western blotting (bottom), and the relative amount of [ $\left.{ }^{3 \mathrm{H}}\right]$ photocholesterolbound receptor versus total receptor was calculated.

SR/CDECL/SR displayed significant ability to bind cholesterol, whereas SR/CDTM/SR did not, which paralleled the findings for eNOS activation (Figure 9, B and C).

\section{Discussion}

The basis for atheroprotection afforded by HDL and SR-BI is poorly understood. In addition to mediating reverse cholesterol transport, HDL initiates signaling in a variety of cell types relevant to atherogenesis (11-14). In the present study, we investigated the proximal events in HDL signaling to eNOS and have demonstrated that HDL-induced cholesterol efflux and multiple domains of SR-BI uniquely mediate signal transduction. Since the intravenous administration of reconstituted HDL restores endothelial function in the brachial artery of hypercholesterolemic patients (33), and intravenous HDL stimulates myocardial perfusion in vivo in wild-type but not eNOS-null mice (34), the initiating events in HDL signaling to eNOS that we have now elucidated result in improved endothelial function and increased coronary blood flow. We postulate that these mechanisms may underlie the reduction in cardiovascular disease risk afforded by HDL.

The role in signal initiation of the well-described capacity of HDL to regulate cholesterol flux was interrogated using multiple strategies. $\mathrm{CD}$ induced eNOS activation, and this response was prevented by cholesterol loading of cyclodextrin. The addition of PC to native HDL, which increases efflux to the lipoprotein (16), yielded an enhanced response. In addition, signaling by native HDL was abrogated by SR-BI neutralizing antibody, which prevents SR-BImediated cholesterol efflux (19). These 3 observations provide strong evidence of a role for efflux in eNOS activation by HDL. Furthermore, cholesterol-free LP2A-I particles caused eNOS acti- vation, whereas cholesterol-containing Lp2A-I did not. These findings also indicate that minor components of $\mathrm{HDL}$, namely estrogen and sphingosine-1-phosphate (S1P), which have been previously implicated in eNOS activation $(35,36)$, are most likely minimally involved in this pathway. This conclusion is consistent with the observations that HDL activates eNOS in cells devoid of estrogen receptors (14) and that it stimulates myocardial perfusion in mice deficient for the $\mathrm{S} 1 \mathrm{P}$ receptor $\mathrm{S}_{1} \mathrm{P}_{3}$ (34). Confirmation of the importance of cholesterol efflux was then obtained in studies of wild-type SR-BI versus the AVI mutant (22), in which the capacity of AVI to mediate efflux to HDL but not to SUVs was paralleled by an ability to signal to eNOS in response to HDL but not to SUV.

Having determined the importance of cholesterol efflux, we then compared signal initiation by SR-BI and CD36. Previous studies indicated that SR-BI mediates eNOS activation by HDL (13). In contrast to SR-BI, CD36, which has similar binding affinity to HDL but induces lower cholesterol efflux to HDL (about $25 \%$ ) (32), failed to stimulate eNOS. Interestingly, SR-BI was also required for signaling in response to $\mathrm{CD}$. These results suggest that in addition to binding HDL, SR-BI senses alterations in the cholesterol environment in the plasma membrane, which leads to the initiation of downstream signals.

In previous work, an SR-BI antibody directed against the C-terminal cytoplasmic domain of the receptor inhibited HDL-mediated eNOS activation in isolated plasma membranes (13). To further investigate the role of the SR-BI C-terminus, we compared signal initiation by SR-BI and by the splice variant SR-BII, which has an alternative C-terminus coded by a downstream exon (23). In contrast to SR-BI, SR-BII was incapable of mediating eNOS activation. Recently the PDZ domain containing protein PDZK1 has 
been shown to interact with the SR-BI C-terminus and play a role in SR-BI cell surface expression in hepatocytes and in SR-BI-mediated selective lipid uptake $(24,37,38)$. PDZK1 is a $70-k D a$ protein comprising $4 \mathrm{PDZ}$ domains without other apparent protein-protein interacting or enzymatic motifs, and it is primarily expressed in kidney, liver, and lung $(37,39)$. Because of the presence of 4 PDZ domains, PDZK1 may act as a scaffold for a macromolecular complex at the plasma membrane. In recent studies, the SR-BI PDZK1-interacting domain was delineated to be the last 3 to 4 C-terminal amino acids of SR-BI (EAKL), which defines a typical class II PDZ domain interaction (24). Furthermore, SR-BI deleted of the last C-terminal leucine (SR-BI $\Delta 509$ ) failed to interact with PDZK1 in vitro (24). In the present work, we found that SR-BI $\Delta 509$ is incapable of mediating eNOS activation by HDL. Thus, PDZK1 or a related protein is likely to be critical to signaling by SR-BI, possibly by serving as a scaffold for the receptor in close proximity to downstream kinases such as the Src family kinases, PI3K, or MAPKs required for eNOS activation by HDL (14). Studies are now warranted to specifically assess PDZK1 involvement in endothelium or alternatively to identify a related endothelial protein coupling SR-BI to downstream signaling molecules.

Having determined that the C-terminal cytoplasmic tail of SR-BI is necessary for signaling by HDL, we performed experiments to assess whether this region is sufficient for mediating these processes. A chimera consisting of CD36 with the C-terminal cytoplasmic domain of SR-BI added to the C-terminus (CD/SRCT; Figure 6) did not cause eNOS phosphorylation or activation, which indicates that the C-terminal cytoplasmic domain of SR-BI is not sufficient to induce HDL signaling. However, a CD36-based chimera with both the SR-BI C-terminal transmembrane and cytoplasmic domains (CD/SRCTMT; Figure 6) displayed eNOS phosphorylation and activation comparable to those of wild-type SR-BI. Thus, both the C-terminal transmembrane and PDZ-interacting domains of SR-BI are required for HDL signaling. In addition, these observations indicate that the level of cholesterol efflux induced by CD36 is sufficient to mediate HDL signaling if the appropriate SR-BI domains are present to transduce signal.

The basis for the requirement for the C-terminal transmembrane domain of SR-BI for signaling was then investigated. The functions of sterol-sensing proteins such as HMG-CoA reductase, SCAP, and NPC1 entail cholesterol binding to transmembrane domains (25-30). We therefore tested the hypothesis that SR-BI also binds membrane cholesterol using a well-characterized photoactivatable, crosslinkable cholesterol analog, photocholesterol. The binding of photocholesterol to SR-BI but not to CD/SRCT was demonstrated, and it was then determined that the C-terminal transmembrane domain of SR-BI is the protein constituent that binds cholesterol. We also found that the third class B scavenger receptor family member, LIMP-II, which is exclusively a resident lysosomal membrane protein, had negligible capacity for cholesterol binding. Furthermore, SR-BI showed greater capacity for cholesterol binding than CD/SRCT or LIMP-II when directed either to the plasma membrane or to lysosomal membranes. This observation indicates that the unique characteristics of the SR-BI C-terminal transmembrane domain, and not attributes specific to the plasma membrane, underlie SR-BI cholesterol binding.

The mechanism(s) by which the C-terminal transmembrane domain of SR-BI binds cholesterol is yet to be determined. The domain does not contain a typical consensus sequence for cholesterol binding, as exists in SCAP, HMG-CoA reductase, and NPC1, in which multiple transmembrane domains mediate the process
(25-29, 40-42). SR-BI also does not share similar secondary or tertiary structure with these proteins. In addition, the newly discovered cholesterol-binding domain of SR-BI lacks the cholesterol recognition/interaction amino acid consensus pattern found in the peripheral-type benzodiazepine receptor and within steroidogenic acute regulatory protein-related (StAR-related) lipid transfer (START) domain sequences $(43,44)$. There are also many other cholesterolbinding proteins including synaptophysins and tetraspanins with noncategorizable cholesterol-binding motifs $(45,46)$. Detailed mutagenesis studies will now be required to elucidate the discrete cholesterol-binding domain of SR-BI. There is evidence that SR-BI may dimerize (47), and therefore C-terminal transmembrane domains of the receptor may act in concert to bind cholesterol.

Furthermore, the mechanistic link between cholesterol binding by the $\mathrm{C}$-terminal transmembrane domain and signal transduction is currently unknown. Recent studies have shown that SCAP undergoes a conformational change upon the addition of cholesterol to endoplasmic reticulum membranes (25). Combining the 3 requirements for signaling revealed in the present work, namely cholesterol efflux, the C-terminal cytoplasmic domain, and the C-terminal transmembrane domain of SR-BI, we postulate that perturbations in cholesterol binding to SR-BI result in conformational change(s) that modify association of the receptor with PDZK1 or other proteins critical to downstream signaling. Additional studies are now warranted to test these possibilities.

In summary, the present work demonstrates that the combination of cholesterol efflux, the SR-BI C-terminal transmembrane domain that binds cholesterol, and the SR-BI C-terminal PDZ-interacting domain are necessary and sufficient for HDL-initiated signaling. These observations provide mechanistic linkage between HDL and the modification of function of endothelial cells and most likely also other cell types involved in atherogenesis. Additionally, they indicate that SR-BI serves as a cholesterol sensor on the plasma membrane. Further work in this area will enhance our fundamental understanding of HDL action and the basis for HDL-related atheroprotection in particular and cholesterol sensing mechanisms in general.

\section{Methods}

Cell culture and transfection. BAECs were prepared and propagated according to standard procedures in EGM-2 media (Biowhittaker Inc.). Experiments were performed at passage 5-8. COS-M6 cells, which express a negligible amount of endogenous SR-BI and are devoid of eNOS, were maintained in DMEM (Sigma-Aldrich) with 10\% fetal calf serum (Invitrogen Corp.). HEK 293 cells were maintained as previously described (48). COS-M6 cells or HEK 293 cells were transfected with various cDNAs using Lipofectamine 2000 (Invitrogen Corp.) according to the manufacturer's instructions. The transfected cells were studied 48 hours after transfection, and $70-80 \%$ transfection efficiency was typically achieved. cDNAs for wild-type eNOS, SR-BI, SR-BII, SR-BIA509, AVI mutant, and chimeras of murine SR-BI and CD36 were prepared as described previously $(9,14,22-24,32)$ (Figure 6). The amino acid delineations of the chimeras were as follows: CD/SRCT, CD36 amino acids 1-458 and SR-BI amino acids 464-509; CD/SRCTMT, CD36 amino acids 1-435 and SR-BI amino acids 436-509; SR/CDECL/SR, CD36 amino acids 44-435 and SR-BI amino acids 1-41 and 436-509; SR/CDTM/SR, SR-BI amino acids 1-435 followed by CD36 amino acids 436-471 and SR-BI amino acids 464-509. In the SR-BI AVI mutant, adenovirus/M45 monoclonal antibody epitopes (14 amino acids) were inserted into a site in the coding sequence of the SR-BI extracellular domain C-terminal to amino acid 424. cDNA for SR-BII was kindly provided by Nancy R. Webb (University of Kentucky, Lexington, Kentucky, USA). It should be noted that CD/SRCT provides full-length 
CD36, which is C-terminally tagged by the addition of a coding sequence for the last 45 amino acids of SR-BI. Because of this, equal expression of different constructs could be confirmed by immunoblot analysis using antibody against the shared C-terminal domain of SR-BI (Novus Biologicals Inc.), and immunoprecipitation was feasible with the same antibody. All functions tested for native $\mathrm{CD} 36$ were recapitulated by $\mathrm{CD} / \mathrm{SRCT}$. We performed additional experiments using cDNA for the lysosomal integral membrane protein LIMP-II, which is highly homologous to CD36 (49), and chimeras of LIMP-II and SR-BI and of LIMP-II and CD36. The C-terminal lysosomal targeting sequence of murine LIMP-II (R456 to T478) was replaced by the last 45 amino acids of SR-BI (LIMP-II/SRCT) to direct LIMP-II to the plasma membrane. We constructed SR-BI/LIMP-II and CD36/LIMP-II chimeras by replacing the lysosomal targeting signal of LIMP-II (R459 to T478) for the last 45 amino acids of SR-BI (SR-BI/LIICT) or for the entire C-terminus of murine CD36 (CD36/LIICT), respectively $(31,50)$. All constructs were generated through PCR and confirmed by sequence analysis. Direct targeting of LIMP-II/SRCT to plasma membranes and SR-BI/LIICT and CD36/LIICT to lysosomal membranes was confirmed by immunofluorescence confocal microscopy.

eNOS activation. eNOS activation in intact primary endothelial cells or transfected COS-M6 cells was assessed by measuring $\left[{ }^{3} \mathrm{H}\right] \mathrm{L}$-arginine (45-70 Ci/mmol, $1 \mathrm{mCi} / \mathrm{ml}$; PerkinElmer Life and Analytical Sciences) conversion to $\left[{ }^{3} \mathrm{H}\right] \mathrm{L}$-citrulline during 15 -minute incubations using previously reported methods (51). The agents employed to test eNOS activation included human HDL, $2 \%$ CD or cholesterol-loaded CD, PC-HDL, SUVs, and LP2A-I particles consisting of recombinant apoA-I and POPC at molar ratios of either $80: 1$ or $40: 1$, with or without cholesterol added at a molar ratio of 5:1 with apoA-I. These agents were prepared as previously described $(13,16-18)$. Of note, Gong and colleagues reported that HDL isolated from female humans or mice has greater capacity to stimulate eNOS than does HDL from male humans or mice, which suggests a role for estradiol associated with HDL (35). However, HDL-mediated responses were apparent at lipoprotein concentrations that would provide estradiol at levels as low as $5 \times 10^{-16} \mathrm{M}(35)$, which is 6 orders of magnitude lower than the concentration shown to activate eNOS in prior reports $(52,53)$, and Nofer and colleagues more recently found no difference in responses to male- versus female-derived HDL (36). Furthermore, we have demonstrated HDL activation of eNOS in a cell system devoid of estrogen receptors (14). Based on these findings, responses to male- versus female-derived HDL were not distinguished in the current experiments. All findings were confirmed in at least 3 independent studies.

Immunoblot analyses. Immunoblot analyses were performed using ECL reagents (Amersham Biosciences) for chemiluminescence. To assess eNOS phosphorylation at Ser1179 in response to HDL, the cells were exposed to HDL $(50 \mu \mathrm{g} / \mathrm{ml})$ for $0-10$ minutes, whole-cell lysates were obtained, and immunoblot analysis was performed with anti-phospho-eNOS polyclonal antibody (Cell Signaling Technology) and anti-eNOS monoclonal antibody (BD Biosciences - Pharmingen). We evaluated expression levels of various SR-BI and CD36 chimeras and SR-BI mutants in whole-cell lysates using SR-BI polyclonal antibodies recognizing C-terminal or extracellular domains of the receptor (Novus Biologicals Inc.). Cell surface expression was assessed by biotinylation, immunoprecipitation with antibody against the SR-BI C-terminus, and probing with streptavidin-HRP conjugate. The expression of LIMP-II-related constructs was assessed following immunoprecipitation by immunoblot analysis with polyclonal anti-LIMP-II antibody generated in rabbit against a peptide corresponding to the last 15 amino acids of murine LIMP-II.

\footnotetext{
1. Brown, M.S., and Goldstein, J.L. 1983. Lipoprotein metabolism in the macrophage: implications for cholesterol deposition in atherosclerosis. Annu. Rev. Biochem. 52:223-261.

2. Gordon, D.J., and Rifkind, B.M. 1989. High-density lipoprotein - the clinical implications of recent
}

Cholesterol efflux. Cholesterol efflux was measured by methods previously described elsewhere (16). Briefly, HEK 293 cells were transfected with wildtype SR-BI or CD36 or chimeras or mutants, cells were labeled for 24 hours with $5 \mu \mathrm{Ci} / \mathrm{ml}\left[{ }^{3} \mathrm{H}\right]$ cholesterol (PerkinElmer Life and Analytical Sciences) in DMEM containing $10 \%$ calf serum, and $\left[{ }^{3} \mathrm{H}\right]$ cholesterol efflux was measured in triplicate during 2-hour incubations with $\operatorname{HDL}(10 \mu \mathrm{g} / \mathrm{ml})$. The release of radioactive cholesterol was measured by scintillation counting.

Photocholesterol binding. To assess the capacity of class B scavenger receptor-related constructs to bind cellular cholesterol, transfected HEK 293 cells or COS-M6 cells were incubated for 16 hours in lipid-free medium containing $\left[{ }^{3} \mathrm{H}\right] 6$-photocholesterol complexed to CD or in $10 \%$ FBS, washed, and ultraviolet irradiated for 20 minutes at room temperature. The cells were lysed, immunoprecipitations were performed using an antibody against the C-terminal tail of SR-BI, and immunoprecipitates were analyzed by SDS-PAGE/fluorography and immunoblot analyses. A comparable approach was recently employed to demonstrate direct binding of cholesterol to SCAP (30). Findings for photocholesterol binding were identical in HEK 293 cells and in COS-M6 cells. Similar strategies were employed following the isolation of plasma membranes or lysosomal membranes to assess cholesterol binding to LIMP-II-related constructs.

Statistical analysis. Differences between groups were evaluated by 1 -way ANOVA after equivalence of variances and normal distribution of data were established. For post-hoc comparisons, we used the Student-Newman-Keuls test. Values shown are mean $\pm \mathrm{SEM} ; n=4$. $P<0.05$ was considered statistically significant.

\section{Acknowledgments}

We are indebted to Marilyn Dixon for preparing this manuscript. This work was supported by NIH grants HL58888 and HL53546 (to P.W. Shaul), HL63768 (to D.L. Williams), and HL22633 and HL63768 (to M. de la Llera-Moya), by grants from the American Heart Association (AHA0130305N to D.L. Silver and 0235107N to C. Mineo), and by a Pfizer International HDL Research Award (CU51610503 to D.L. Silver). The project was also supported in part by the Crystal Charity Ball Center for Pediatric Critical Care Research and by the Lowe Foundation.

Received for publication November 8, 2004, and accepted in revised form February 2, 2005.

Address correspondence to: Philip W. Shaul, Department of Pediatrics, University of Texas Southwestern Medical Center at Dallas, 5323 Harry Hines Boulevard, Dallas, Texas 75390, USA. Phone: (214) 6482015; Fax: (214) 648-2481; E-mail: Philip.Shaul@utsouthwestern. edu. Or to: David L. Silver, Department of Medicine, Division of Molecular Medicine, Columbia University College of Physicians and Surgeons, New York, New York 10032, USA. Phone: (212) 342-1320; Fax: (212) 305-5052; E-mail: dls51@Columbia.edu.

Chatchawin Assanasen and Chieko Mineo contributed equally to this work.

Philip W. Shaul and David L. Silver are co-senior authors.

David L. Williams is deceased.

studies. N. Engl. J. Med. 321:1311-1316.

3. Stein, O., and Stein, Y. 1999. Atheroprotective mechanisms of HDL. Atherosclerosis. 144:285-301.

4. Trigatti, B., Rigotti, A., and Krieger, M. 2000.

The role of the high-density lipoprotein receptor SR-BI in cholesterol metabolism. Curr. Opin. Lipidol.
11:123-131.

5. Calvo, D., and Vega, M.A. 1993. Identification, primary structure, and distribution of CLA-1, a novel member of the CD36/LIMPII gene family. J. Biol. Chem. 268:18929-18935.

6. Acton, S.L., Scherer, P.E., Lodish, H.F., and Krieger, 
M. 1994. Expression cloning of SR-BI, a CD36related class B scavenger receptor. J. Biol. Chem. 269:21003-21009.

7. Llera-Moya, M., et al. 1999. Scavenger receptor BI (SR-BI) mediates free cholesterol flux independently of HDL tethering to the cell surface. J. Lipid Res. 40:575-580.

8. Kellner-Weibel, G., et al. 2000. Expression of scavenger receptor BI in COS-7 cells alters cholesterol content and distribution. Biochemistry. 39:221-229.

9. Connelly, M.A., et al. 2001. Analysis of chimeric receptors shows that multiple distinct functional activities of scavenger receptor, class B, type I (SR-BI), are localized to the extracellular receptor domain. Biochemistry. 40:5249-5259.

10. Jolley, C.D., Woollett, L.A., Turley, S.D., and Dietschy, J.M. 1998. Centripetal cholesterol flux to the liver is dictated by events in the peripheral organs and not by the plasma high density lipoprotein or apolipoprotein A-I concentration. J. Lipid Res. 39:2143-2149.

11. Grewal, T., et al. 2003. High density lipoproteininduced signaling of the MAPK pathway involves scavenger receptor type BI-mediated activation of Ras. J. Biol. Chem. 278:16478-16481.

12. Nofer, J.R., et al. 2002. HDL and arteriosclerosis: beyond reverse cholesterol transport. Atherosclerosis. 161:1-16.

13. Yuhanna, I.S., et al. 2001. High-density lipoprotein binding to scavenger receptor-BI activates endothelial nitric oxide synthase. Nat. Med. 7:853-857.

14. Mineo, C., Yuhanna, I.S., Quon, M.J., and Shaul, P.W. 2003. High density lipoprotein-induced endothelial nitric-oxide synthase activation is mediated by Akt and MAP kinases. J. Biol. Chem. 278:9142-9149.

15. Blair, A., Shaul, P.W., Yuhanna, I.S., Conrad, P.A., and Smart, E.J. 1999. Oxidized low density lipoprotein displaces endothelial nitric-oxide synthase (eNOS) from plasmalemmal caveolae and impairs eNOS activation. J. Biol. Chem. 274:32512-32519.

16. Yancey, P.G., et al. 2000. High density lipoprotein phospholipid composition is a major determinant of the bi-directional flux and net movement of cellular free cholesterol mediated by scavenger receptor BI. J. Biol. Chem. 275:36596-36604.

17. Meng, Q.H., Sparks, D.L., and Marcel, Y.L. 1995 Effect of LpA-I composition and structure on cholesterol transfer between lipoproteins. J. Biol. Chem. 270:4280-4287.

18. Sparks, D.L., Frank, P.G., Braschi, S., Neville, T.A., and Marcel, Y.L. 1999. Effect of apolipoprotein A-I lipidation on the formation and function of pre-beta and alpha-migrating LpA-I particles. Biochemistry. 38:1727-1735.

19. Gu, X., Kozarsky, K., and Krieger, M. 2000. Scavenger receptor class B, type I-mediated [3H]cholesterol efflux to high and low density lipoproteins is dependent on lipoprotein binding to the receptor. J. Biol. Chem. 275:29993-30001.

20. Jian, B., et al. 1998. Scavenger receptor class B type I as a mediator of cellular cholesterol efflux to lipoproteins and phospholipid acceptors. J. Biol. Chem. 273:5599-5606.

21. Kilsdonk, E.P., et al. 1995. Cellular cholesterol efflux mediated by cyclodextrins. J. Biol. Chem. 270:17250-17256.
22. Connelly, M.A., et al. 2003. Separation of lipid transport functions by mutations in the extracellular domain of scavenger receptor class B, type I. J. Biol. Chem. 278:25773-25782.

23. Webb, N.R., et al. 1998. SR-BII, an isoform of the scavenger receptor BI containing an alternate cytoplasmic tail, mediates lipid transfer between high density lipoprotein and cells. J. Biol. Chem. 273:15241-15248.

24. Silver, D.L. 2002. A carboxyl-terminal PDZ-interacting domain of scavenger receptor $B$, type $I$ is essential for cell surface expression in liver. J. Biol. Chem. 277:34042-34047.

25. Brown, A.J., Sun, L., Feramisco, J.D., Brown, M.S., and Goldstein, J.L. 2002. Cholesterol addition to ER membranes alters conformation of SCAP, the SREBP escort protein that regulates cholesterol metabolism. Mol. Cell. 10:237-245.

26. Carstea, E.D., et al. 1997. Niemann-Pick C1 disease gene: homology to mediators of cholesterol homeostasis. Science. 277:228-231.

27. Hua, X., Nohturfft, A., Goldstein, J.L., and Brown, M.S. 1996. Sterol resistance in $\mathrm{CHO}$ cells traced to point mutation in SREBP cleavage-activating protein. Cell. 87:415-426.

28. Loftus, S.K., et al. 1997. Murine model of NiemannPick C disease: mutation in a cholesterol homeostasis gene. Science. 277:232-235.

29. Nohturfft, A., Brown, M.S., and Goldstein, J.L. 1998. Topology of SREBP cleavage-activating protein, a polytopic membrane protein with a sterolsensing domain. J. Biol. Chem. 273:17243-17250.

30. Adams, C.M., et al. 2004. Cholesterol and 25hydroxycholesterol inhibit activation of SREBPs by different mechanisms, both involving SCAP and insigs. J. Biol. Chem. 279:52772-52780.

31. Tabuchi, N., Akasaki, K., and Tsuji, H. 2002. Ile (476), a constituent of di-leucine-based motif of a major lysosomal membrane protein, LGP85/LIMP II, is important for its proper distribution in late endosomes and lysosomes. Biochem. Biophys. Res. Commun. 295:149-156.

32. Connelly, M.A., Klein, S.M., Azhar, S., Abumrad, N.A., and Williams, D.L. 1999. Comparison of class B scavenger receptors, CD36 and scavenger receptor $\mathrm{BI}$ (SR-BI), shows that both receptors mediate high density lipoprotein-cholesteryl ester selective uptake but SR-BI exhibits a unique enhancement of cholesteryl ester uptake. J. Biol. Chem. 274:41-47.

33. Spieker, L.E., et al. 2002. High-density lipoprotein restores endothelial function in hypercholesterolemic men. Circulation. 105:1399-1402.

34. Levkau, B., et al. 2004. High-density lipoprotein stimulates myocardial perfusion in vivo. Circulation. 110:3355-3359.

35. Gong, M., et al. 2003. HDL-associated estradiol stimulates endothelial NO synthase and vasodilation in an SR-BI-dependent manner. J. Clin. Invest. 111:1579-1587. doi:10.1172/JCI200316777.

36. Nofer, J.R., et al. 2004. HDL induces NO-dependent vasorelaxation via the lysophospholipid receptor S1P3. J. Clin. Invest. 113:569-581. doi:10.1172/ JCI200418004.

37. Ikemoto, M., et al. 2000. Identification of a PDZdomain-containing protein that interacts with the scavenger receptor class B type I. Proc. Natl. Acad.
Sci. U. S. A. 97:6538-6543.

38. Kocher, O., et al. 2003. Targeted disruption of the PDZK1 gene in mice causes tissue-specific depletion of the high density lipoprotein receptor scavenger receptor class B type I and altered lipoprotein metabolism. J. Biol. Chem. 278:52820-52825.

39. Wang, S., Yue, H., Derin, R.B., Guggino, W.B., and $\mathrm{Li}, \mathrm{M}$. 2000. Accessory protein facilitated CFTR-CFTR interaction, a molecular mechanism to potentiate the chloride channel activity. Cell. 103:169-179.

40. Radhakrishnan, A., Sun, L.P., Kwon, H.J., Brown, M.S., and Goldstein, J.L. 2004. Direct binding of cholesterol to the purified membrane region of SCAP: mechanism for a sterol-sensing domain. Mol. Cell. 15:259-268

41. Mann, R.K., and Beachy, P.A. 2000. Cholesterol modification of proteins. Biochim. Biophys. Acta. 1529:188-202.

42. Kuwabara, P.E., and Labouesse, M. 2002. The sterol-sensing domain: multiple families, a unique role? Trends Genet. 18:193-201.

43. Li, H., and Papadopoulos, V. 1998. Peripheral-type benzodiazepine receptor function in cholesterol transport. Identification of a putative cholesterol recognition/interaction amino acid sequence and consensus pattern. Endocrinology. 139:4991-4997.

44. Ponting, C.P., and Aravind, L. 1999. START: a lipidbinding domain in StAR, HD-ZIP and signalling proteins. Trends Biochem. Sci. 24:130-132.

45. Thiele, C., Hannah, M.J., Fahrenholz, F., and Huttner, W.B. 2000. Cholesterol binds to synaptophysin and is required for biogenesis of synaptic vesicles. Nat. Cell Biol. 2:42-49.

46. Charrin, S., et al. 2003. A physical and functional link between cholesterol and tetraspanins. Eur. J. Immunol. 33:2479-2489.

47. Reaven, E., Cortez, Y., Leers-Sucheta, S., Nomoto, A., and Azhar, S. 2004. Dimerization of the scavenger receptor class B type I: formation, function, and localization in diverse cells and tissues. J. Lipid Res. 45:513-528.

48. Silver, D.L., Wang, N., and Vogel, S. 2003. Identification of small PDZK1-associated protein, DD96/MAP17, as a regulator of PDZK1 and plasma high density lipoprotein levels. J. Biol. Chem. 278:28528-28532.

49. Vega, M.A., et al. 1991. Cloning, sequencing, and expression of a cDNA encoding rat LIMP II, a novel 74-kDa lysosomal membrane protein related to the surface adhesion protein CD36. J. Biol. Chem. 266:16818-16824.

50. Eskelinen, E.L., Tanaka, Y., and Saftig, P. 2003. At the acidic edge: emerging functions for lysosomal membrane proteins. Trends Cell Biol. 13:137-145.

51. Pace, M.C., et al. 1999. Establishment of an immortalized fetal intrapulmonary artery endothelial cell line. Am. J. Physiol. 277:L106-L112.

52. Lantin-Hermoso, R.L., et al. 1997. Estrogen acutely stimulates nitric oxide synthase activity in fetal pulmonary artery endothelium. Am. J. Physiol. 273:L119-L126.

53. Caulin-Glaser, T., Garcia-Cardena, G., Sarrel, P., Sessa, W.C., and Bender, J.R. 1997. 17 beta-estradiol regulation of human endothelial cell basal nitric oxide release, independent of cytosolic $\mathrm{Ca} 2+$ mobilization. Circ. Res. 81:885-892. 